\title{
MODELAGEM DA VULNERABILIDADE DOS AQUÍFEROS NO MUNICÍPIO DE ARACATU - SUDOESTE DO ESTADO DA BAHIA
}

\author{
MAIA, P. H.P. ${ }^{1 *}$; CRUZ, M. J. M. ${ }^{2} \&$ RODRIGUES, R. S. ${ }^{3}$ \\ 1 - Diretoria de Monitoramento, Setor de Hidrogeologia, INGÁ- Inst. de Gestão das Águas \\ 2 - Departamento de Geoquímica, Instituto de Geociências, Universidade Federal da Bahia \\ 3 - Universidade Salvador- UNIFACS \\ *Corresponding author: phpmaia@gmail.com
}

\begin{abstract}
Maia, P. H.P.; Cruz, M. J. M. \& Rodrigues, R. S. (2013) Vulnerability modelling for aquifers in Aracatu county - southwestern Bahia state. Braz. J. Aquat. Sci. Technol. 17(1):53-60. DOI:10.14210/bjast.v17.n1.p53-60. elSSN 1983-9057. To ensure the sustainable use of water and adequate protection of the aquifer is necessary to know the areas most vulnerable to contamination and establish programs specific inspection and monitoring. The objective of this research was to model the vulnerability of aquifers in the county of Aracatu using methods DRASTIC, GOD and MAIA, in order to compare the results obtained. This paper presents the results of mapping the vulnerability of aquifers in the county of Aracatu, located in the southwestern state of Bahia in semi-arid region, where the population lives with recurrent droughts and water shortages of good quality. The region is located in south-central part of São Francisco Craton, where crystalline rocks outcrop of Archean and Proterozoic age with a variety of structural alignments, predominantly in the direction NW-SE and NE-SW, parallel to the main fracture systems that aquifer system is in a fissure of great importance for rural supply, especially in dry periods, when groundwater is the only alternative to supplying the population. The vulnerability indices were calculated and modeled using the software Surfer 8 . 0. The vulnerability indices were correlated with each other and the concentrations of nitrate, using maps and charts to evaluate the behavior of each.
\end{abstract}

Keywords: Modelling, inherent vulnerability, aquifers.

\section{INTRODUÇÃO}

Distante cerca de $600 \mathrm{~km}$ da capital do estado, o municípiode Aracatu encontra-se totalmente inserido na região Semi-árida (SUDENE, 2005). A população do município convive com secas recorrentes e escassez de água de boa qualidade.

Do ponto de vista geotectônico, a área está situada na porção centro-sul do Cráton de São Francisco (Almeida, 1977), onde predominam rochas de idade Proterozóica inferior e Arqueanas, sobre as quais efeitos tectônicos e/ou metamórficos levaram a profundas modificações de ordem lito-estrutural. Os alinhamentos estruturais possuem direções NW-SE e NE-SW, predominantemente, paralelos aos principais sistemas de fraturas. Também ocorrem faixas estreitas e isoladas de sequências vulcanosedimentares do denominado Bloco do Gavião (Maia, 2011).

Os aquíferos da região, predominantemente fissurais, apresentam solos pouco espessos e níveis estáticos rasos. Mais de dez poços apresentam na análise de suas águas valores de nitritos acima do máximo permitido para fins de potabilidade e estas concentrações elevadas de nitrito indicam tratar-se de contaminação recente (Maia et al., 2009).

Apesar das águas serem salobras, parte delas apresentarem constituintes potencialmente prejudiciais com teores acima do permitido, elas se constituem em uma alternativa segura de abastecimento nos períodos secos (Maia, 2005). A vulnerabilidade intrínseca de um aquífero é decorrente das propriedades físico-químicas inerentes do meio aquífero e que expressa a susceptibilidade hidrogeológica da água ser afetada por contaminantes (Auge, 2003).

Em função da necessidade de proteção das águas subterrâneas, observa-se um incremento de modelos e técnicas de mapeamento de vulnerabilidade à poluição de aquíferos, principalmente nas últimas três décadas, constituindo uma importante ferramenta na gestão racional das águas subterrâneas. A cartografia de vulnerabilidade tem servido a racionalizar ações de proteção de aquíferos, na medida em que tenta compatibilizar as atividades antrópicas com a capacidade do terreno em suportá-las, sem prejuízo das águas subterrâneas (Hirata, 2001).

Encontram-se na bibliografia sobre o assunto, diversos métodos de avaliação da vulnerabilidade do aquífero à contaminação (Foster et al., 2001), que podem ser classificados em três grupos principais, de acordo com a abordagem adotada por Vrba \& Zaporozec (1994):

a) Ambientes hidrogeológicos: baseiam a avaliação da vulnerabilidade, em termos qualitativos, nas características gerais do ambiente, usando mapas temáticos (Albinet \& Margat, 1970).

b) Modelos análogos: utilizam expressões matemáticas para os parâmetros essenciais tais como 
tempo de trânsito médio na zona vadosa como indicadores do índice de vulnerabilidade (Monkhouse, 1983).

c) Sistemas paramétricos: usam parâmetros selecionados como indicadores de vulnerabilidade e aplicam seu espectro de valores e interações para produzir alguma forma de índice de vulnerabilidade relativo ou absoluto. Incluem como exemplo desse método o EPIK, desenvolvido especificamente para aquíferos cársticos e discutido por Doerfliger e Zwahlen (1997); o DRASTIC de Aller et al. (1987); o GOD de Foster (1987) e outro método digno de nota nessa categoria é o MAIA de Maia et al. (2011).

Alguns desses sistemas incluem índices de vulnerabilidade formados por parâmetros hidrogeológicos, morfológicos e outras formas de parametrização das características dos aquíferos de um modo bem definido. A adoção de índices de vulnerabilidade tem a vantagem, em princípio, de eliminar ou minimizar a subjetividade inerente aos processos de avaliação.

A vulnerabilidade de um aquífero é caracterizada por meio dos seguintes fatores naturais:

a) acessibilidade da zona saturada à penetração de poluentes,

b) capacidade de atenuação resultante da retenção físico-química ou reação ao poluente na zona não saturada e,

c) a diluição e a remobilização dos contaminantes.

A atuação desses fatores pode ser avaliada com a aplicação em uma equação matemática que traduz um índice de vulnerabilidade a partir de variáveis que representam características intrínsecas do meio aquífero, nos tres métodos avaliados (Foster et al., 2001).

Os fatores que influenciam o comportamento hidrogeológico dos aquíferos variam em função da intensidade do fraturamento nos aquíferos fissurais, do grau de carstificação nos cársticos e nos granulares dos ambientes deposicionais e processos diagenéticos envolvidos. Esses fatores não são avaliados nos métodos tradicionais que quantificam a vulnerabilidade natural dos aquíferos, uma vez que nesses métodos as variáveis utilizadas são determinadas por meio de tabelas e vinculadas direta e indiretamente a litologia dos aquíferos, o que provoca a diferença observada entre os índices calculados pelos diferentes métodos.

Todos os aquíferos são vulneráveis, a médio ou a longo prazo, a contaminantes que apresentam características persistentes e móveis, gerados por uma atividade amplamente distribuída em uma região. A carga poluidora pode ser controlada ou modificada, o mesmo não ocorre com a vulnerabilidade natural, que é uma propriedade intrínseca do aquífero (Foster et al.,1988).

À exceção do EPIK, específico para aquíferos carsticos, os principais métodos utilizados para deteminar a vulnerabilidade de aquíferos foram desenvol- vidos para aquiferos granulares e todos determinam índices qualitativos.

No Congresso da IAH realizado na Bratislava em 1999, "ocorreram divergências entre as escolas Alemã e Checa: a primeira sustentava a necessidade de novas tecnologias para transformar a vulnerabilidade em uma variável quantitativa e, a escola Checa propunha a manutenção do alcance qualitativo, devido ao "inconveniente" de se atribuir grandezas aos componentes e processos que incidem sobre a vulnerabilidade" (Auge, 2004).

Com o advento dos sistemas SIG, existe uma tendência a que se produzam métodos quantitativos para a definição da vulnerabilidade intrínseca dos aquíferos (Auge, 2004). O método MAIA (Maia \& Cruz, 2011) foi desenvolvido neste contexto está sendo colocado como uma alternativa para a modelagem da vulnerabilidade de aquíferos, tanto localmente como a nível regional.

O objetivo desta pesquisa foi o de modelar a vulnerabilidade dos aquíferos no município de Aracatu (BA) utilizando os métodos DRASTIC, GOD e MAIA, com a finalidade de comparar os índices obtidos, correlacionando-os às concentrações de nitrato observadas nas águas subterrâneas.

\section{MATERIAIS E MÉTODOS}

A área estudada está localizada no sudoeste do Estado da Bahia, na bacia hidrográfica do rio de Contas em uma sub-bacia de seu afluente, rio Gavião. Possui uma área de $1541,5 \mathrm{~km}^{2}$ e a sede do município está situada em $14^{\circ} 25^{\prime} 00^{\prime \prime}$ e e $41^{\circ} 27^{\prime} 00^{\prime \prime} W$ em uma altitude de $720 \mathrm{~m}$ acima do nível do mar (Figura 1).

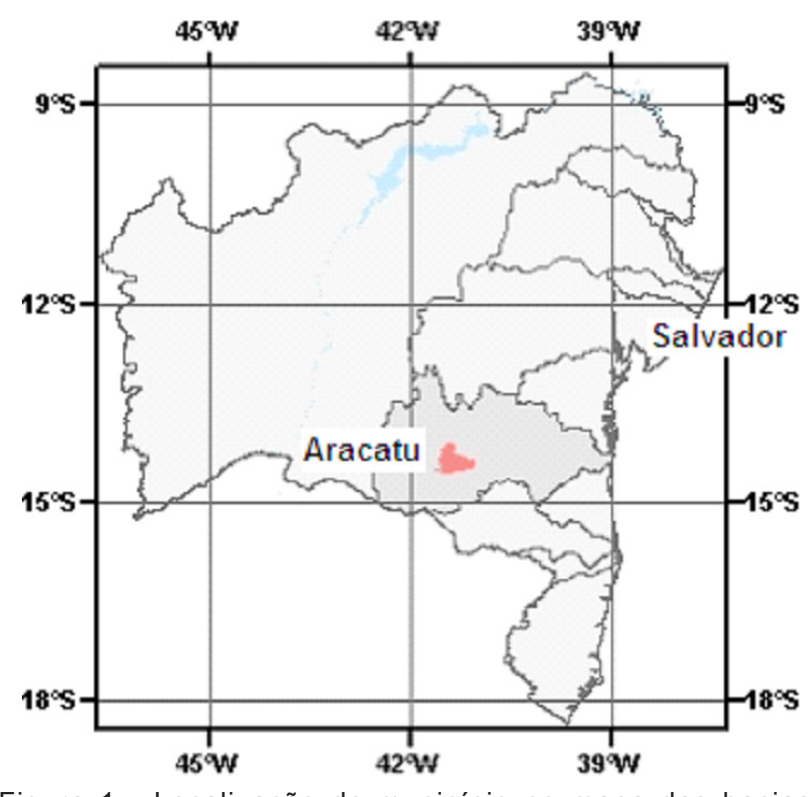

Figura 1 - Localização do município no mapa das bacias hidrográficas. 
O presente trabalho se constituí na complementação da dissertação de mestrado desenvolvida por um dos autores (Maia, 2005), cujos dados foram utilizados para a determinação da vulnerabilidade natural dos aquíferos na área, pelos métodos citados anteriormente.

De modo geral, os métodos tradicionais para avaliar a vulnerabilidade foram desenvolvidos, para um tipo particular de aquífero, quase sempre definindo os valores das variáveis a serem empregadas no cálculo da vulnerabilidade, por meio de tabelas e aplicando fatores de ponderação definidos com bastante subjetividade a cada uma delas, o que compromete seus resultados.

Com os dados obtidos a partir das tabelas de aplicação dos métodos tradicionais (para detalhes sobre o modo de coleta dos dados ver Maia \& Cruz, 2011), juntamente com as variáveis medidas no sistema aquífero, foram construídas planilhas no software Microsoft Excel para serem processadas e aplicadas nas equações de cada método para determinar os índices de vulnerabilidade (Tabela 1).

Os valores dos índices de vulnerabilidade calculados para cada um dos métodos foram espacializados com o método de interpolação krigagem com configurações default (Jakob, 2002), que utiliza o dado tabular e sua posição geográfica para calcular as interpolações de dados dispersos e pouco abundantes. Para tanto foi empregado o software Surfer 8.0, que gerou os mapas com os índices de vulnerabilidade DRASTIC, GOD e MAIA.
Os mapas de cada um dos índices foram correlacionados, por meio de mapas e gráficos, com as concentrações de nitrato, de maneira a possibilitar a avaliação do desempenho e limitações de cada um dos métodos.

\section{RESULTADOS}

\section{Cartografia da Vulnerabilidade com o Método DRASTIC}

Os valores das variáveis requisitadas para aplicação do método DRASTIC foram atribuídos a partir da tabela que orienta sua aplicação, considerando os dados dos poços para em seguida, serem calculados por meio da equação, os índices de vulnerabilidade (Tabela 2).

Observa-se que ocorre uma repetição nos valores das variáveis, pois estas são obtidas por meio de tabelas, vinculadas diretamente a litologia do aquífero ou indiretamente, como o solo derivado. Este procedimento produz uma tendência de equalizar os valores gerando poucos índices de vulnerabilidade.

Os valores dos índices calculados foram espacializados, utilizando o software Surfer 8.0 gerando o mapa de vulnerabilidade (Figura 2).

O mapa com as concentrações de nitrato foi produzido utilizando o resultado das análises das águas efetuadas após a perfuração dos poços (Maia, 2005).

A correlação entre os indices Drastic e as concentrações de nitrato revela a ocorrencia de uma faixa

Tabela 1 - Equações e variáveis dos Métodos: GOD, DRASTIC e MAIA.

\begin{tabular}{ll}
\hline \hline Índice & Parâmetros \\
\hline & Índice = G. O. D \\
sendo \\
(G) grau de confinamento \\
(O) litologia e o grau de consolidação da cobertura \\
(D) profundidade da superfície freática (Foster, 1987). \\
Índice = D. Dw + R. Rw + A. Aw + S. Sw + T. Tw + I. Iw + C. Cw \\
sendo \\
(D) profundidade da superfície freática \\
(R) taxa de recarga \\
(A) tipo do aqǘfero \\
(S) tipo de solo \\
(T) topografia ou declividade do terreno \\
(I) impacto da zona não saturada \\
(C) condutividade hidráulica \\
respectivos fatores de ponderação: Dw=5 e, Rw=4, Aw=3, Sw=2, Tw=1, Iw=5 e \\
Cw=3, (Aller et al., 1987). \\
V= [(1/PA + 1/ES + 1/DT) + CE + RE + TA ou DF ] \\
sendo \\
(PA) profundidade da água \\
(ES) espessura do solo \\
(DT) declividade do terreno \\
(CE) capacidade específica \\
(RE) recarga \\
(TA) transmissividade do aquífero ou (DF) densidade de fraturas (Maia et al., 2011) \\
\hline \hline
\end{tabular}


Tabela 2 - Cálculo do Índice DRASTIC, para o município de Aracatu.

\begin{tabular}{|c|c|c|c|c|c|c|c|c|c|c|c|c|c|c|c|c|c|c|c|}
\hline Poço & Localidade & $\overline{\mathbf{X}}$ & $\overline{\mathbf{Y}}$ & D & Dw & إR & Rw & $\mathbf{A}$ & Aw & $\mathbf{S}$ & Sw & $\bar{T}$ & $\overline{T w}$ & 1 & Iw & C & CWw & Índice & Qualificação \\
\hline 1 & Meleiro & $-14,47806$ & $-41,53694$ & 10 & 5 & 1 & 4 & 3 & 3 & 9 & 2 & 10 & 1 & 4 & 5 & 1 & 3 & 114,00 & Muito baixa \\
\hline 2 & Faz. Fundão & $-14,43250$ & $-41,34056$ & 9 & 5 & 1 & 4 & 3 & 3 & 9 & 2 & 1 & 1 & 4 & 5 & 1 & 3 & 100,00 & Insignificante \\
\hline 3 & Baixa Escura & $-14,37500$ & $-41,29111$ & 9 & 5 & 1 & 4 & 3 & 3 & 9 & 2 & 9 & 1 & 4 & 5 & 1 & 3 & 108,00 & Muito baixa \\
\hline 4 & Pimenta & $-14,54750$ & $-41,43500$ & 5 & 5 & 1 & 4 & 3 & 3 & 9 & 2 & 10 & 1 & 4 & 5 & 1 & 3 & 89,00 & Insignificante \\
\hline 5 & Faz. Pau Ferro & $-14,38889$ & $-41,30806$ & 9 & 5 & 3 & 4 & 3 & 3 & 9 & 2 & 5 & 1 & 4 & 5 & 1 & 3 & 112,00 & Muito baixa \\
\hline 6 & Faz. Manoel Velho & $-14,45639$ & $-41,33806$ & 9 & 5 & 3 & 4 & 3 & 3 & 7 & 2 & 9 & 1 & 4 & 5 & 1 & 3 & 112,00 & Muito baixa \\
\hline 7 & Faz. Duas Irmãs & $-14,30250$ & $-41,32361$ & 9 & 5 & 1 & 4 & 3 & 3 & 9 & 2 & 9 & 1 & 4 & 5 & 1 & 3 & 108,00 & Muito baixa \\
\hline 8 & Algodão & $-14,41667$ & $-41,38500$ & 5 & 5 & 1 & 4 & 3 & 3 & 10 & 2 & 10 & 1 & 4 & 5 & 2 & 3 & 94,00 & Insignificante \\
\hline 9 & Sede III & $-14,41667$ & $-41,48250$ & 10 & 5 & 1 & 4 & 3 & 3 & 10 & 2 & 9 & 1 & 4 & 5 & 1 & 3 & 115,00 & Muito baixa \\
\hline 10 & Pé de Serra & $-14,42611$ & $-41,22694$ & 1 & 5 & 3 & 4 & 3 & 3 & 9 & 2 & 9 & 1 & 4 & 5 & 1 & 3 & 76,00 & Insignificante \\
\hline 11 & Sede V & $-14,40528$ & $-41,45833$ & 10 & 5 & 1 & 4 & 3 & 3 & 9 & 2 & 9 & 1 & 4 & 5 & 1 & 3 & 113,00 & Muito baixa \\
\hline 12 & Eixo Serra II - Lg Represa & $-14,26000$ & $-41,44417$ & 9 & 5 & 1 & 4 & 3 & 3 & 9 & 2 & 10 & 1 & 4 & 5 & 1 & 3 & 109,00 & Muito baixa \\
\hline 13 & Passagem do Lagedo II & $-14,37194$ & $-41,56528$ & 9 & 5 & 1 & 4 & 3 & 3 & 9 & 2 & 10 & 1 & 4 & 5 & 1 & 3 & 109,00 & Muito baixa \\
\hline 14 & Patos & $-14,21194$ & $-41,53389$ & 7 & 5 & 1 & 4 & 3 & 3 & 9 & 2 & 9 & 1 & 4 & 5 & 1 & 3 & 98,00 & Insignificante \\
\hline 15 & Zacarias & $-14,44583$ & $-41,22222$ & 10 & 5 & 3 & 4 & 3 & 3 & 7 & 2 & 9 & 1 & 4 & 5 & 1 & 3 & 117,00 & Muito baixa \\
\hline 16 & Lagoa de Santana & $-14,33056$ & $-41,21250$ & 9 & 5 & 3 & 4 & 3 & 3 & 9 & 2 & 9 & 1 & 4 & 5 & 1 & 3 & 116,00 & Muito baixa \\
\hline 17 & Várzea da Pedra & $-14,27139$ & $-41,34611$ & 10 & 5 & 1 & 4 & 3 & 3 & 9 & 2 & 5 & 1 & 4 & 5 & 1 & 3 & 109,00 & Muito baixa \\
\hline 18 & Serra Negra & $-14,27528$ & $-41,42111$ & 9 & 5 & 1 & 4 & 3 & 3 & 9 & 2 & 10 & 1 & 4 & 5 & 2 & 3 & 112,00 & Muito baixa \\
\hline 19 & Caetano & $-14,27417$ & $-41,38500$ & 10 & 5 & 1 & 4 & 3 & 3 & 9 & 2 & 3 & 1 & 4 & 5 & 1 & 3 & 107,00 & Muito baixa \\
\hline 20 & Paris do Matias & $-14,16278$ & $-41,44583$ & 9 & 5 & 1 & 4 & 3 & 3 & 7 & 2 & 10 & 1 & 4 & 5 & 1 & 3 & 105,00 & Insignificante \\
\hline 21 & Várzea da Pedra VI & $-14,27361$ & $-41,17139$ & 10 & 5 & 1 & 4 & 3 & 3 & 9 & 2 & 10 & 1 & 4 & 5 & 1 & 3 & 114,00 & Muito baixa \\
\hline 22 & Várzea da Pedra III & $-14,27389$ & $-41,17111$ & 5 & 5 & 1 & 4 & 3 & 3 & 9 & 2 & 5 & 1 & 4 & 5 & 1 & 3 & 84,00 & Insignificante \\
\hline
\end{tabular}

de vulnerabilidade insignificante, com índices que variam de 76-105 a oeste e a nordeste, sem ocorrência de nitratos e outra área localizada a leste, com altas concentrações de nitratos. Tambem ocorre uma faixa de vulnerabilidade muito baixa localizada o norte e sudeste, com índices variando 107-117, onde ocorrem concentrações de nitrato tanto baixas como altas (Figura 2 e Figura 3). A correlação do índice DRASTIC com a concentração de nitratos, por meio de mapas, revela que os índices obtidos não estão coerentes nos locais onde o contaminante foi detectado nas águas subterrâneas (Figura 3).

\section{Cartografia da Vulnerabilidade com o Método GOD}

Os valores das variáveis requisitadas para a modelagem da vulnerabilidade com o método GOD, obtidos a partir dos dados dos poços e do diagrama do método, foram aplicados na equação produzindo os índices abaixo (Tabela 3). Aqui também ocorre uma repetição nos valores das variáveis, pois de três duas delas são obtidas por meio de tabelas, vinculadas diretamente a litologia do aquífero produzindo uma tendência gerar índices com valores muito próximos.

Os valores dos índices calculados foram espacializados utilizando o software Surfer 8.0 gerando o mapa de vulnerabilidade GOD (Figura 4).

A correlação com a concentração de Nitratos evidencia setores com vulnerabilidade Média coincidentes com áreas contaminadas a Norte e a Leste do município e áreas sem contaminação a Oeste e, locais com Baixa vulnerabilidade como o setor central de direção SW-NE, coincidente com uma faixa com teores elevados de Nitratos (Figura 3).

Tabela 3 - Cálculo do Índice GOD, para o município de Aracatu.

\begin{tabular}{lccccccccc}
\hline \hline Poço N & Numero & Localidade & $\mathbf{X}$ & $\mathbf{Y}$ & $\mathbf{G}$ & $\mathbf{O}$ & $\mathbf{D}$ & Índice & Qualificação \\
\hline $3.044 / 85$ & 1 & Meleiro & $-14,47806$ & $-41,53694$ & 0,6 & 0,4 & 0,9 & 0,216 & Baixa \\
3. 220/85 & 2 & Faz. Fundão & $-14,43250$ & $-41,34056$ & 0,6 & 0,4 & 0,9 & 0,216 & Baixa \\
$2.352 / 84$ & 3 & Baixa Escura & $-14,37500$ & $-41,29111$ & 0,6 & 0,6 & 0,9 & 0,324 & Média \\
$4.469 / 88$ & 4 & Pimenta & $-14,54750$ & $-41,43500$ & 0,6 & 0,4 & 0,8 & 0,192 & Baixa \\
$4162 / 87$ & 5 & Faz. Pau Ferro & $-14,38889$ & $-41,30806$ & 0,6 & 0,4 & 0,9 & 0,216 & Baixa \\
$4157 / 87$ & 6 & Faz. Manoel Velho & $-14,45639$ & $-41,33806$ & 0,6 & 0,4 & 0,9 & 0,216 & Baixa \\
$4147 / 87$ & 7 & Faz. Duas Irmãs & $-14,30250$ & $-41,32361$ & 0,6 & 0,4 & 0,9 & 0,216 & Baixa \\
$3.139 / 85$ & 8 & Algodão & $-14,41667$ & $-41,38500$ & 0,6 & 0,8 & 0,8 & 0,384 & Média \\
$2.830 / 85$ & 9 & Sede III & $-14,41667$ & $-41,48250$ & 0,6 & 0,7 & 0,9 & 0,378 & Média \\
$2.358 / 84$ & 10 & Pé de Serra & $-14,42611$ & $-41,22694$ & 0,6 & 0,4 & 0,7 & 0,168 & Baixa \\
$4.482 / 88$ & 11 & Sede V & $-14,40528$ & $-41,45833$ & 0,6 & 0,7 & 0,9 & 0,378 & Média \\
$2-1712$ & 12 & Eixo Serra II & $-14,26000$ & $-41,44417$ & 0,6 & 0,7 & 0,9 & 0,378 & Média \\
15942 & 13 & Passagem do Lagedo II & $-14,37194$ & $-41,56528$ & 0,6 & 0,6 & 0,9 & 0,324 & Média \\
$2-1707$ & 14 & Patos & $-14,21194$ & $-41,53389$ & 0,6 & 0,8 & 0,8 & 0,384 & Média \\
1720 & 15 & Zacarias & $-14,44583$ & $-41,22222$ & 1 & 0,4 & 0,9 & 0,360 & Média \\
$4154 / 87$ & 16 & Lagoa de Santana & $-14,33056$ & $-41,21250$ & 0,6 & 0,7 & 0,9 & 0,378 & Média \\
$2.355 / 84$ & 17 & Várzea da Pedra & $-14,27139$ & $-41,34611$ & 0,6 & 0,4 & 0,9 & 0,216 & Baixa \\
$2.243 / 84$ & 18 & Serra Negra & $-14,27528$ & $-41,42111$ & 0,6 & 0,7 & 0,9 & 0,378 & Média \\
$2-1733$ & 19 & Caetano & $-14,27417$ & $-41,38500$ & 0,6 & 0,7 & 0,9 & 0,378 & Média \\
21728 & 20 & Paris do Matias & $-14,16278$ & $-41,44583$ & 0,6 & 0,4 & 0,9 & 0,216 & Baixa \\
1-6491 & 21 & Várzea da Pedra VI & $-14,27361$ & $-41,17139$ & 0,6 & 0,7 & 0,9 & 0,378 & Média \\
$5.113 / 92$ & 22 & Várzea da Pedra III & $-14,27389$ & $-41,17111$ & 0,6 & 0,4 & 0,8 & 0,192 & Baixa \\
\hline \hline
\end{tabular}




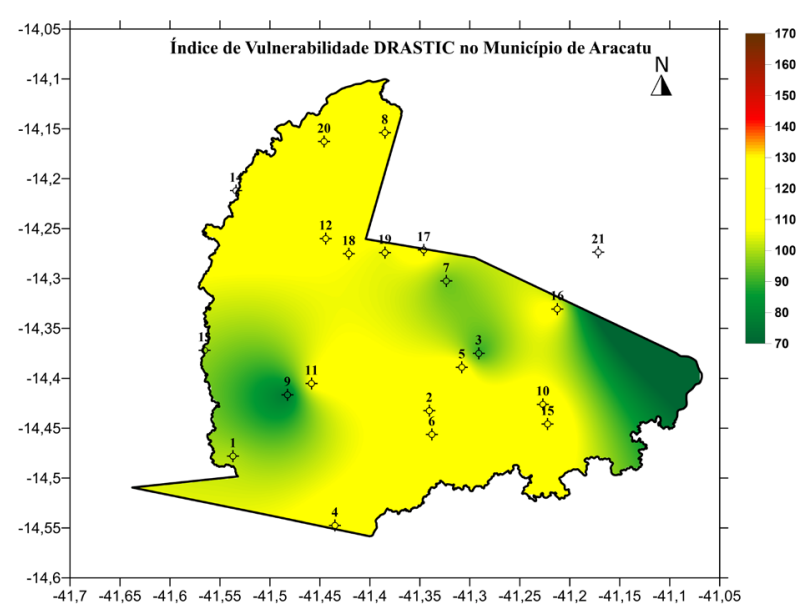

Figura 2 - Mapa dos índices de vulnerabilidade DRASTIC no município de Aracatu.

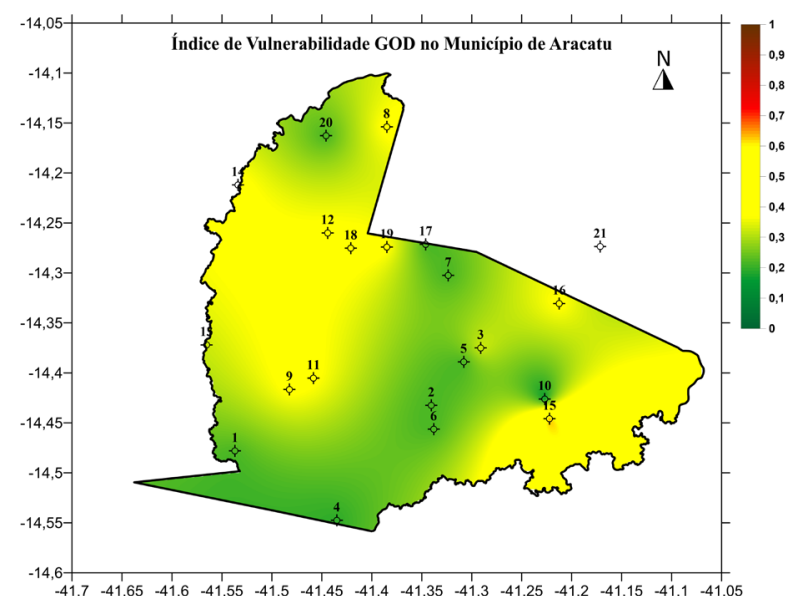

Figura 4 - Mapa dos índices de vulnerabilidade GOD no município de Aracatu.

\section{Cartografia da Vulnerabilidade com o Método MAIA}

As variáveis requisitadas pelo método MAIA, todas efetivamente medidos nos perfis dos poços e no terreno, foram aplicados na equação produzindo os índices apresentados na Tabela 4. Observa-se que estes estão vinculados somente aos valores das variáveis medidas no aquífero e em o seu entorno, com reflexos sobre a sensibilidade do método em detectar pequenas variações nos parâmetros.

Os valores dos índices calculados foram espacializados, utilizando o software Surfer 8.0, gerando o mapa com os índices de vulnerabilidade MAIA (Figura 5). Pode-se destacar os índices baixos no setor oeste do município, com direção NNE, gradando para índices de vulnerabilidade média na mesma direção no setor central, até atingir os índices mais altos no setor Leste.

A comparação do mapa dos índices de vulnerabilidade MAIA com o mapa da distribuição de Nitratos, revela uma boa correlação entre as concentrações elevadas do contaminante e as áreas de média e alta vulnerabilidade. No setor central do município a faixa

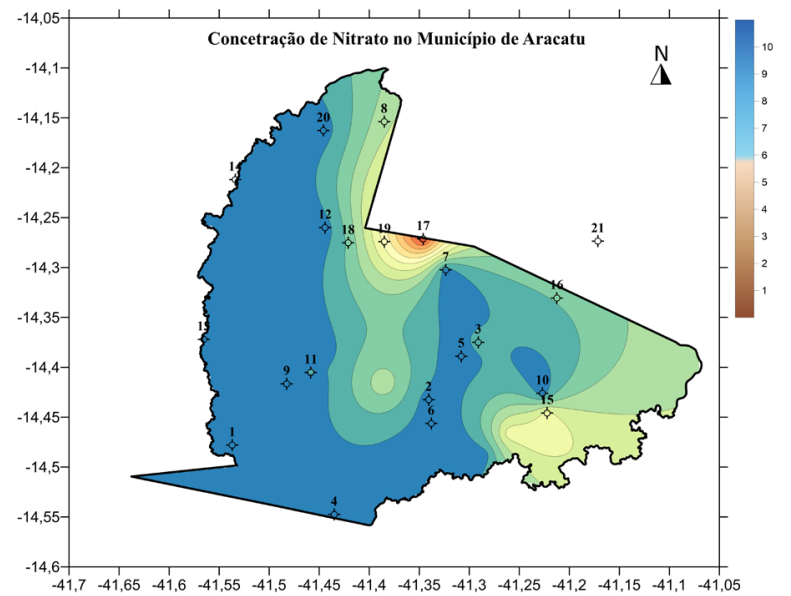

Figura 3 - Mapa das concentrações de nitrato no município de Aracatu.

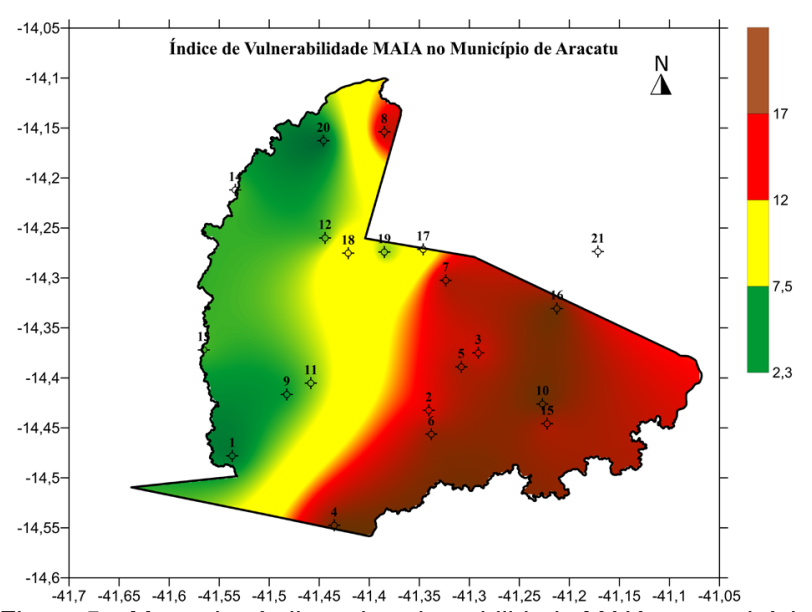

Figura 5 - Mapa dos índices de vulnerabilidade MAIA no município de Aracatu.

com concentrações elevadas de Nitrato com direção NE-SW, coincide com os locais onde a vulnerabilidade é média. Aárea localizada a SE-NW as concentrações elevadas ocorrem onde os índices de vulnerabilidade são altos (Figura 3 e Figura 5).

Apesar dos resultados obtidos com a aplicação do método MAIA, estes devem ser visto com ressalvas. É possível um aquífero se configurar uma situação de alta vulnerabilidade, porém sem risco de poluição devido à ausência de carga poluidora ou ao contrário, configurar uma situação de baixa vulnerabilidade com risco elevado pela presença de uma carga poluidora significativa. Entretanto os índices de vulnerabilidade calculados estão coerentes com a distribuição da pluviosidade que corresponde a recarga potencial (Figura 6) e com a topografia observada no modelo numérico do terreno (Figura 7). 


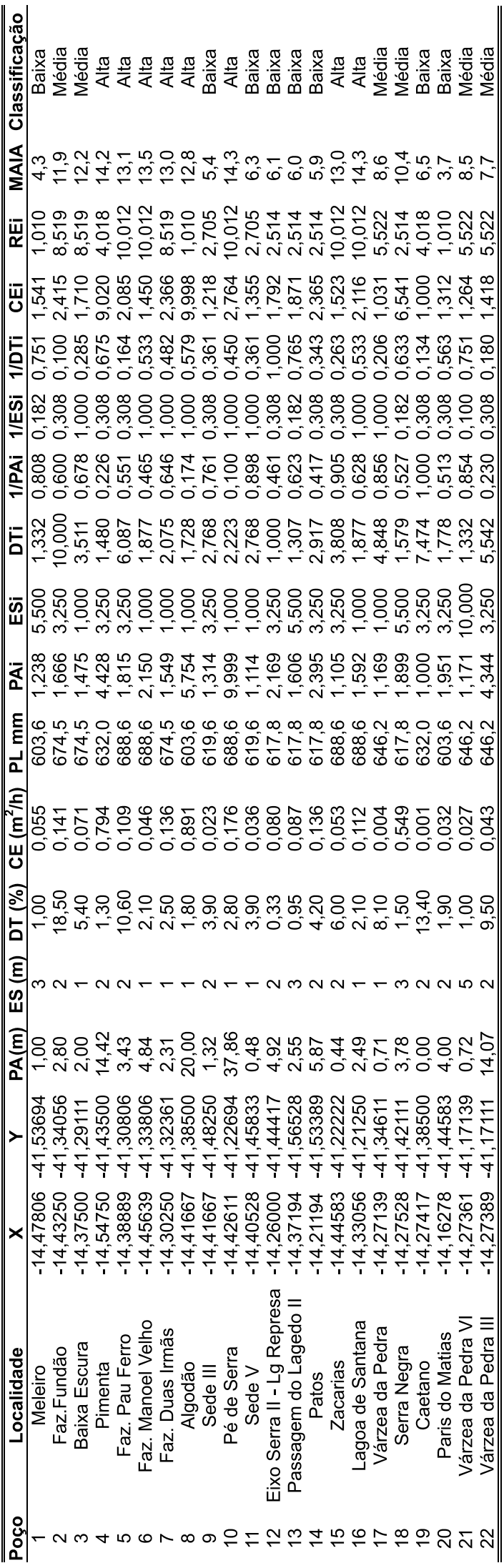

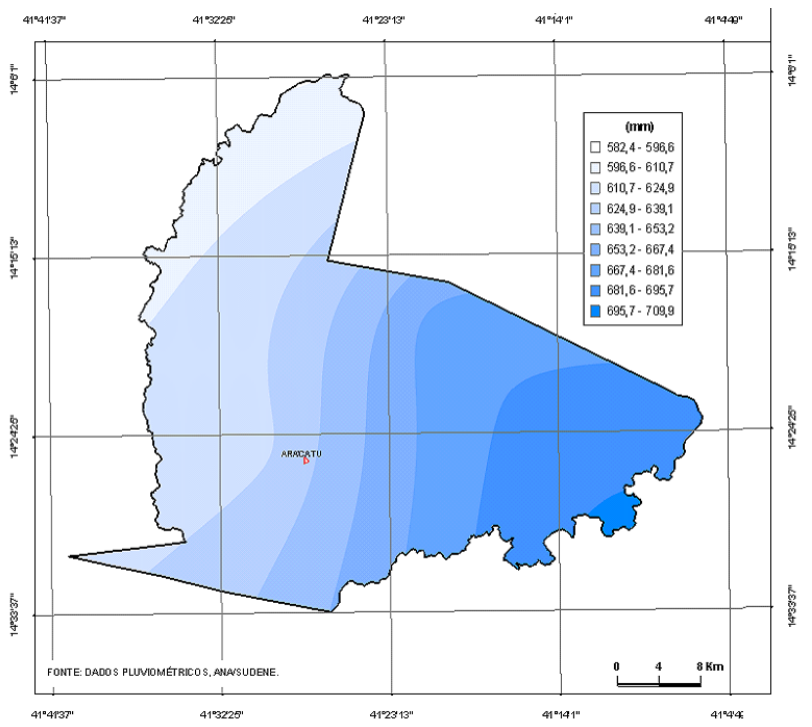

Figura 11 - Distribuição da pluviosidade no município de Aracatu.

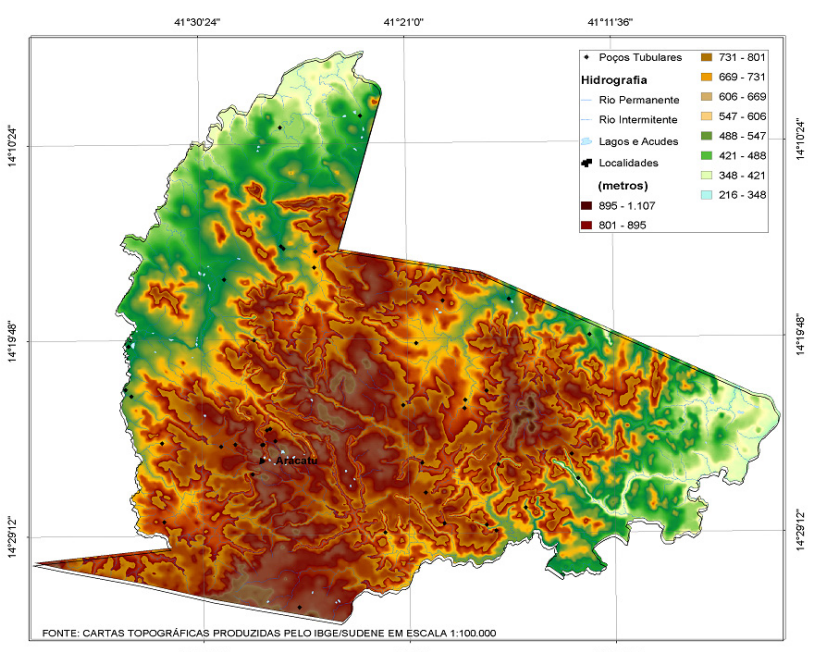

Figura 12- Modelo numérico de elevação do terreno no município de Aracatu. Fonte: Maia (2005).

\section{DISCUSSÃO}

As variáveis do DRASTIC que dependem da litologia, como: Natureza do Aquífero (A) e Impacto da Zona não Saturada (I), determinados por meio de tabelas, apresentam os mesmos valores em quase todos os poços. A Recarga Líquida (R) e a Permeabilidade (C) também apresentam valores repetidos. Isso faz com que os índices de vulnerabilidade determinados pelo método sejam iguais ou muito parecidos.

Os índices de vulnerabilidade DRASTIC variaram de 76 a 117, em decorrência da repetição dos valores das variáveis vinculadas a rochas e solos que produziram somente duas classes de vulnerabilidade: insignificante e predominantemente muito baixa, caracterizando os aquíferos de Aracatu como bem 
Tabela 5 - Correlação dos índices de vulnerabilidade com Nitratos.

\begin{tabular}{cc}
\hline \hline $\begin{array}{c}\text { Índices de } \\
\text { Vulnerabilidade }\end{array}$ & $\begin{array}{c}\text { Coeficientes de Correlação } \\
\text { com Nitratos }\end{array}$ \\
\hline MAIA & 0,0029 \\
GOD & 0,0015 \\
DRASTIC & 0,0244 \\
\hline \hline
\end{tabular}

protegidos. Esse resultado provavelmente é consequência da forma como se determinam os valores das variáveis (Tabela 2).

O índice de vulnerabilidade GOD variou de 0,168 a 0,384 provavelmente devido à repetição dos valores das variáveis, sendo que duas das três que compõe o método são vinculadas a litologia dos aquíferos. Também produziram somente duas classes de vulnerabilidade, apresentando índices predominantemente Baixos e Médios.

Esses aquíferos submetidos a grandes esforços tectônicos apresentam fraturas expostas na superfície do terreno, solos pouco espessos e níveis d'agua relativamente rasos, sendo bastante vulneráveis. Entretanto apresentaram índices de vulnerabilidade DRASTIC variando de Insignificante a Muito Baixo, e índices GOD predominantemente Baixo a Médio. Assim ambos os métodos qualificam os aquíferos do município como bem protegidos.

Os coeficientes de correlação entre os índices de vulnerabilidade dos três métodos aplicados $\left(R^{2}\right)$ e as concentrações de Nitrato no município de Aracatu foram calculados sendo muito baixos nos três métodos (Tabela 5), denotando que não existe relação matemática entre eles.

Atualmente não se conhece um método descrito na literatura cujos índices de vulnerabilidade apresentem um coeficiente de correlação razoável com as concentrações de Nitrato e, de acordo com Auge (2003), a solubilidade, mobilidade e persistência de certos contaminantes como os nitratos, fazem com que algumas variáveis percam consistência em relação à vulnerabilidade.

A equação descreve a vulnerabilidade a partir dos efeitos de proteção representados pelas parcelas: Profundidade da Água (PA), Espessura do Solo (ES) e Declividade Topográfica (DT), inversamente relacionadas a atenuação e acessibilidade a contaminantes, Adicionado aos efeitos produzidos na vulnerabilidade pelas parcelas: Capacidade Específica (CE), Recarga (RE) e Densidade de Fraturas (DF) ou Transmissividade do Aquífero (TA), diretamente relacionadas a acessibilidade de contaminantes.

A matriz de correlação entre os índices de vulnerabilidade MAIA com os índices do DRASTIC e do GOD aponta para coeficientes menores que 0,3576 que traduz uma total falta de relação entre eles, provavelmente em decorrência da utilização de diferentes variáveis em equações distintas.

\section{CONCLUSÕES}

Decorrente da vinculação de algumas variáveis à litologia da tabela, em áreas com mesmo tipo rocha e de solo, sem grandes variações nos níveis d'água, os métodos DRASTIC e o GOD tendem a produzir variáveis repetidas, índices próximos e poucas classes de vulnerabilidade.

O fato é que esses métodos além de terem sido desenvolvidos para aplicações em aquíferos granulares, a forma de obtenção das variáveis por meio de tabelas e o uso de fatores de ponderação aumenta a possibilidade de se obter índices similares por meio de uma combinação de diferentes situações hidrogeológicas. Isto compromete os resultados obtidos no cálculo da vulnerabilidade e pode acarretar em riscos na tomada de decisões.

O mapa do índice de vulnerabilidade MAIA apresenta valores baixos no setor oeste do município, variando de 4,29 a 6,46, com direção NNE, gradando para valores dos índices de vulnerabilidade médios, variando de 7,66 a 12,19, na mesma direção no setor central, até atingir os índices mais altos, variando de 12,76 a 14,33, no setor leste.

É notável a correlação por meio de mapas do índice MAIA com a distribuição de nitratos no município de Aracatu que evidencia uma concentração de valores mais elevados na porção central, com direção NE-SW, e no setor leste do município, coincidentes com áreas onde os índices de vulnerabilidade variam de médio a alto, com índices de vulnerabilidade mais baixos a oeste

Apesar deste resultado corroborar para a validação do método, ele deve ser visto com ressalvas, pois um aquífero pode configurar uma situação de alta vulnerabilidade, porém sem risco de poluição devido a ausência de carga poluidora ou ao contrário, configurar uma situação de baixa vulnerabilidade com risco elevado pela presença de uma carga poluidora significativa.

O método Maia apresenta características de um modelo análogo e paramétrico que utiliza uma expressão algébrica constituída por parcelas de variáveis independentes indicadoras de vulnerabilidade. A estratégia adotada no seu desenvolvimento foi a de usar somente variáveis mensuráveis que, além de imprimir um caráter universal ao método, acentua a sensibilidade do índice para detectar diferenças decorrentes de pequenas variações nos parâmetros. 


\section{REFERENCIAS}

Albinet, M. \& Margat, J. 1970. Cartogrrafhie de La Vulnerabilite a La Poluition des Nappes d'eau Soterraine. Bull. BRGM, 2me Series, 3(4): 13-22.

Aller, L. \& Bennet, T. 1987. DRASTIC: a standardized system for evaluating groundwater pollution potential using hydrological settings. U. S. Environmental Protection Agency, Ada, OK, EPA, Report 600/2-87-035; 455p.

Almeida, F. F. M. 1997. Craton do São Francisco. Rev. Bras. Geociências 7(4): 349-364.

Auge, M. 2003. Vulnerabilidad de acuíferos. Conceptos y métodos. Ebook: 1-38. RedIRIS Red Académica y Científica de España en Internet.

Auge, M. 2004. Vulnerabilidade de aquíferos. Rev. Lat. -Am. Hidrogeo. 4: 85-103.

Doerfliger, N. \& Zwahlem, F. 1997. Epik A new method for outlining of protection areas in karstic environment. Proceedings of the Int. Symp. on Karst Waters and Environ. Impacts [s. n. ], 117-123pp.

Foster S. S. D. 1987. Fundamental concepts in aquifer vulnerability, pollution risk and protection strategy. Vulnerability of Soil and Groundwater to Pollutants. TNO Committee on Hydrological Research Information, 38p.

Foster, S. S. D.; Hirata, R. C. A. \& Rocha, G. A. 1988. Riscos de poluição de água subterrânea: uma proposta de avaliação regional. CEPIS/OPS, São Paulo.

Foster, S. S. D.; Hirata, R. C. A.; Gomes, D.; D'elia, M. \& Paris, M. 2001. Groundwater quality protection: a guide for water service companies, municipal authorities and environment agencies. Washington, D. C; Banco Mundial.

Hirata, R. C. A. 2001. Oito perguntas e oito tentativas de respostas sobre a Vulnerabilidade à poluição de aquífero. In: I Seminario-Taller. Protección de Acuíferos Frente a La Contaminación, São Paulo. Disponível em <http://tierra. rediris. es/hidrored/ ponencias/Hirata. html>.

Jakob, A. A. E. 2002. Kriging demographic variables in order to look for trends in the spatial distribution of population. Spatial Modeling and Analysis CSS 620, Department of Crop and Soil Sciences, Cornell University, Ithaca, NY.

NRC (National Research Council). 1993. Groundwater vulnerability assessment: contamination potential under conditions of uncertainty. National Academy Press, Washington, D. C..

Monkhouse, R. A. 1983. Vulnerability of aquifers and groundwater quality in the United Kingdom. Institute of Geological Sciences Report, Nottingham, Reino Unido.

Paralta, E., Frances, A. \& Ribeiro, L. 2005. Avaliação da Vulnerabilidade do Sistema Aquífero dos Gabros de Beja e Análise Crítica das redes de monitorização no Contexto da Directiva Quadro da Água. Publicações do $7^{\circ}$ Simpósio de Hidráulica e recursos Hídricos dos Países de Língua Oficial Portuguesa (SISLUBA). Évora.

Maia, P. H. P. 2005. Meio ambiente e potencialidades hídricas na região de Aracatu, sudoeste da Bahia. Dissertação de Mestrado, Universidade Federal da Bahia - UFBA. 191 pp.

Maia, P. H. P.; Cruz, M. J. M. \& Sampaio, M. C. 2009. Zoneamento dos aquíferos do Estado da Bahia. Braz. J. Aq. Sci. Tech. 13(1): 45-52.

Maia, P. H. P. \& Cruz, M. J. M. 2011. Um Novo Método para Avaliar a Vulnerabilidade de Aquíferos. Braz. J. Aq. Sci. Tech. 15(2): 29-40.

Maia, P. H. P Um novo método para avaliar a vulnerabilidade dos aquíferos. Tese de Doutorado, Instituto de Geociências da Universidade Federal da Bahia, Salvador, 2011. 130pp.

SUDENE, 2005. Portaria $\mathrm{n}^{\circ} 89$ de 16 de março de 2005 que atualiza a relação dos municipios pertencentes a região Semi-Árida do Fundo Constitucional de Financiamento do Nordeste-FNE.

Vrba, J. \& Zaporozec, A. 1994. Guidebook on mapping groundwater vulnerability. Hannover: International Association of Hydrogeologists. 131pp.

Submetido: Dezembro/2011 Revisado: Outubro/2012 Aceito: Setembro/2013 